\title{
Software libre aplicado al dibujo industrial: el caso Blender
}

\begin{abstract}
RESUMEN
El presente artículo pretende dar respuesta a la siguiente pregunta problemática ¿cómo mantener actualizado un laboratorio de dibujo industrial en el tema de software, considerando las restricciones económicas que pueda tener una universidad? Para eso se verifican diversas alternativas de software libre y se analiza la implementación de uno llamado Blender. Los resultados se traducen en un alto grado de aceptación del software por parte de los alumnos, esto se puede apreciar en los diversos comentarios $y$ resultados de sus evaluaciones.
\end{abstract}

Palabras Clave: Software libre, Blender, dibujo industrial, licencia, propiedad intelectual.

FREE SOFTWARE APPLIED TO INDUSTRIAL DRAWING: THE BLENDER CASE

\section{ABSTRACT}

The article pretends to give an answer to the following problematic question how to maintain up to date an industrial drawing laboratory in software considering the economics restrictions that can be a university? For this, it review free software alternatives and analyze the implementation of one of them call Blender. The results it traduce in a high acceptation grade of this software in the students, it can see in the students comment and results of their tests.

KEYwoRDs: Free software, Blender, Industrial drawing, License, intellectual propriety.

\section{INTRODUCCIÓN}

En la actualidad es cotidiano el uso de software en los diversos procesos tanto académicos como laborales; su aprendizaje constituye una herramienta fundamental para todo profesional o personal técnico. Dentro del diseño industrial existen diversas alternativas, las cuales tienen ventajas en cuanto a su uso; sin embargo, y sobre todo para el ámbito académico, su principal dificultad está en el precio que se debe pagar por la adquisición de las licencias. Esta situación, en muchos casos, los hace prácticamente inaccesibles para diversas universidades por lo que se perjudica el alumnado y se elitiza cada vez más el proceso de enseñanza y aprendizaje.

El objetivo del presente artículo es mostrar una experiencia práctica de implementación de un software libre como complemento al dictado del curso de dibujo industrial, haciendo una evaluación preliminar de su resultado e impacto en los alumnos del curso; asimismo, se pretende incentivar en los docentes que estén afrontando un problema similar al descrito en el resumen, la búsqueda de soluciones mediante software libre.

\section{TIPOS DE SOFTWARES UTILIZADOS EN EL DIBUJO INDUSTRIAL}

Entre los tipos de softwares utilizados en la actualidad existen diversas clasificaciones, sin embargo, en forma general se pueden mencionar la siguiente:

\section{Software en $2 d$ para la construcción de planos}

Este tipo de software es muy útil para la construcción de planos de piezas industriales, viviendas, instalaciones industriales e incluso se puede considerar a aquellos softwares especializados en el patronaje textil. Muchos de estos softwares son parte de un modelo en tres dimensiones, es decir, tienen un módulo que puede soportar esta construcción.

\section{Software para modelamiento}

Este tipo de software es muy útil para la elaboración de una pieza virtual en tres dimensiones, pues permite apreciar cómo es

1 Magíster en ingeniería industrial. Profesor del Departamento de Diseño y Tecnología Industrial, UNMSM. E-mail: achungp@unmsm.edu.pe 
que se verá la pieza una vez terminada; como se deduce del caso anterior este software puede tener un módulo para la generación del plano de la pieza modelada. Se recomienda considerar las opciones de acabado final de la pieza virtual y el juego de luces y sombras que el software provea, debido a que esto le dará un mayor realismo al diseño.

\section{Software para animación}

El software para animación permite generar una secuencia de movimientos de una pieza en un escenario modelado; es muy útil para ilustrar ensambles de maquinaria, diagramas de explosión, paseos virtuales, entre otros. Se recomienda tomar en cuenta las consideraciones del Software para Modelamiento detallados en el punto anterior.

\section{Software para simulación}

Este tipo de software recrea las condiciones a las cuales va a estar sometida una determinada pieza a fin de evaluar su resistencia, entre otros factores.

Blender se encuentra en la clasificación 2 y 3 de la lista anteriormente mencionada.

La tendencia que están experimentando los softwares industriales relacionados al diseño es hacia la Integración, en este caso se está refiriendo a dos integraciones. La primera CAD/CAM, si bien esta tendencia ya se está dando, es de esperarse que se acentúe cada vez más sobre todo dentro de lo que es el CAE. La segunda integración está referida a los cuatro tipos de clasificaciones anteriormente mencionados.

Con respecto a Blender se puede decir que existe un script aún en fase de pre-alfa llamado Blender$\mathrm{Cad}^{2}$, el cual pretende entrar en el proceso de integración mencionado.

\section{SOFTWARE LIBRE}

Se define como software libre aquel software que permite a los usuarios ejecutar, copiar, distribuir, estudiar, cambiar y mejorar el software sin restricción alguna por motivo de licencias u otros; es decir, debe cumplir con lo que se conoce como las cuatro libertades fundamentales ${ }^{3}$ del software libre:

- La libertad de usar el programa, con cualquier propósito (libertad 0).
- La libertad de estudiar cómo funciona el programa y adaptarlo a sus necesidades (libertad 1). El acceso al código fuente es una condición previa para esto.

- La libertad de distribuir copias, con lo que se puede ayudar a otro usuario (libertad 2).

- La libertad de mejorar el programa y hacer públicas las mejoras a los demás, de modo que toda la comunidad se beneficie (libertad 3). El acceso al código fuente es un requisito previo para esto.

Así pues, el concepto de software libre no se restringe únicamente al hecho de que sea gratuito (sobre todo si se piensa en tal y como se escribe en inglés "free software") sino que es un asunto de "libertad", es decir, libertad en el uso, estudio o modificación del software, lo cual implica el acceso al conocimiento que el software encierra sin las restricciones de las licencias de usuario.

El uso de software libre está reglamentado por la Licencia Pública General también conocida como GNU-GPL por haber sido elaborada por el proyecto GNU; esta licencia se puede considerar como una "anti licencia" con respecto a las licencias tradicionales del software comercial, esto se debe a que contrariamente a una licencia tradicional, esta licencia no restringe sino más bien permite las cuatro libertades anteriormente mencionadas.

Sin embargo, cabe mencionar que la GNU-GPL no es la única licencia existente en el tema de software libre pero es la más conocida.

Poco a poco, el uso del software libre se está generalizando cada vez más en diversas instituciones, entre ellas las universidades; como ejemplo se puede mencionar el caso de la Facultad de Ingeniería Industrial de la Universidad Nacional Mayor de San Marcos, la cual luego del decreto respectivo ha mudado sus sistemas a software libre.

El software libre se ha convertido rápidamente en una alternativa en diferentes áreas de trabajo, quizás el uso más conocido es como aulas virtuales con el software MOODLE ${ }^{4}$, por otro lado también hay que mencionar la alternativa al paquete OFFICE como es OPEN OFFICE ${ }^{5}$, entre otros.

Existe pues una contradicción entre el software comercial y el software libre, mientras que los pri-

\footnotetext{
Para mayor información consultar la página web: http://projects.blender.org/projects/blendercad/

Para mayor información consultar la página web del proyecto GNU: www.gnu.org

Para mayor información consultar: moodle.org

Para mayor información consultar: www.openoffice.org
} 
meros plantean el derecho de restringir el uso de su "propiedad intelectual" (lo cual es un derecho legítimo), este derecho excluiría a todas aquellas instituciones o personas que no puedan pagar los altos derechos de uso de aprender y actualizarse en el manejo del software, convirtiéndolos en analfabetos informáticos.

\section{EL SOFTWARE LIBRE EN EL CAMPO DEL DIBUJO INDUSTRIAL}

Dentro del campo del dibujo industrial, en especial en el modelamiento en tres dimensiones y en el movimiento, existen diversas alternativas en software libre, como por ejemplo Wings 3D, el cual es un potente generador de imágenes en tres dimensiones. Sin embargo, no genera movimiento, al menos hasta la versión 0.99. La Figura 1 presenta una muestra de la galería de su página web ${ }^{6}$.

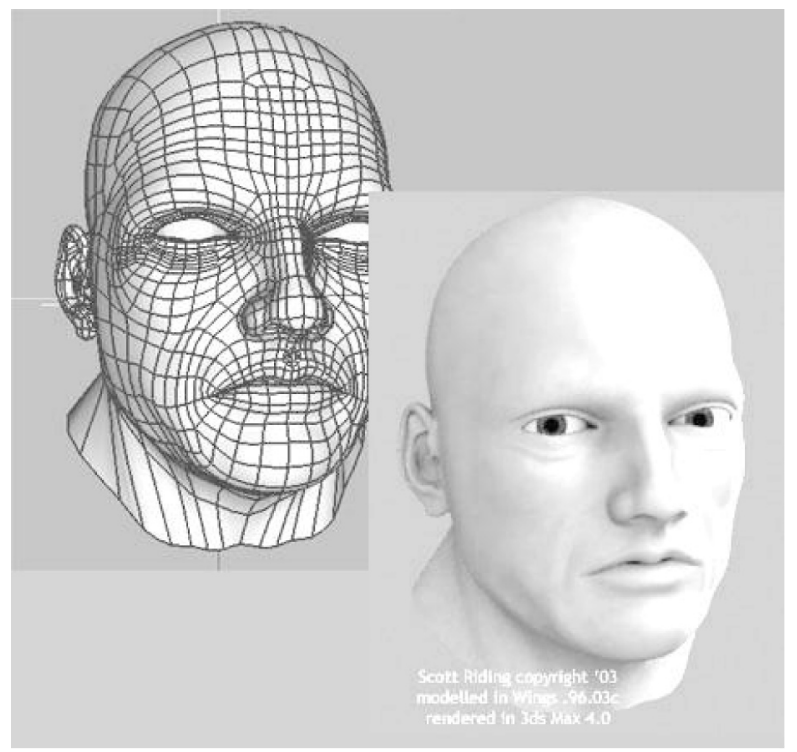

Figura 1. Muestra de Wings 3D.

Otro ejemplo a examinar es un software libre desarrollado por la armada norteamericana, el cual se llama BRL-CAD ${ }^{7}$; similar al caso anterior, este programa puede realizar diversas construcciones tridimensionales, sin embargo, aún no puede realizar movimiento; la Figura 2 muestra una parte de la galería de su página web.

También cabe mencionar el software Art of Illusion ${ }^{8}$, cuyos resultados presenta una alta calidad, sobre todo en el renderizado; para tener una mejor idea véase la Figura 3.

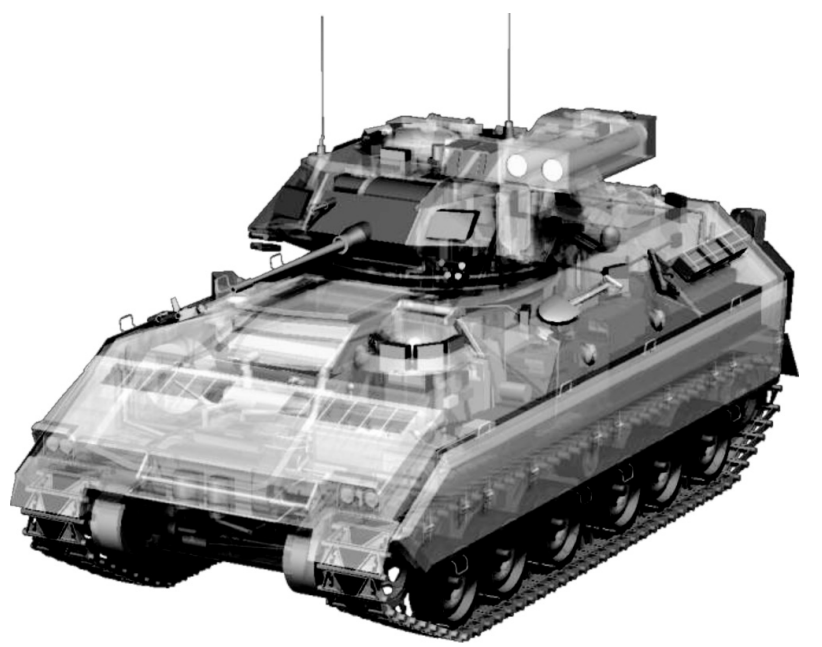

Figura 2. Muestra del software BRL-CAD.

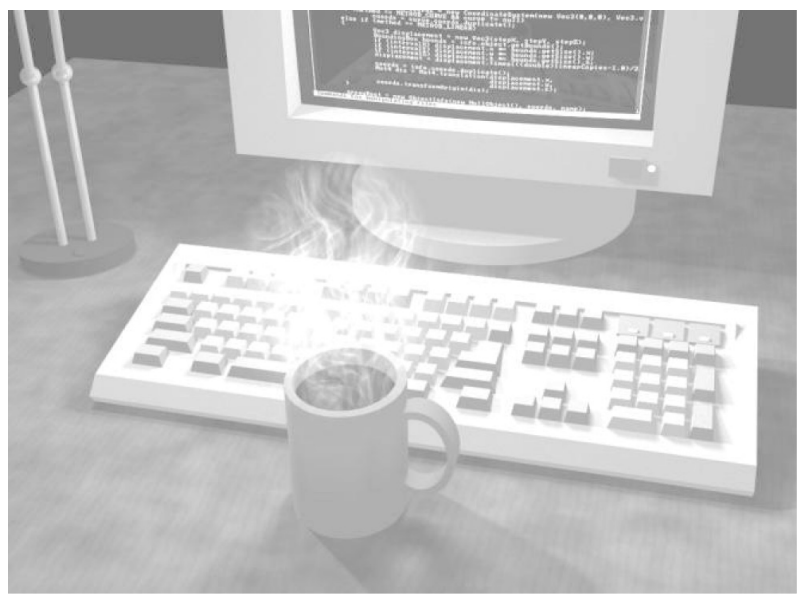

Figura 3. Muestra de Art of llusion.

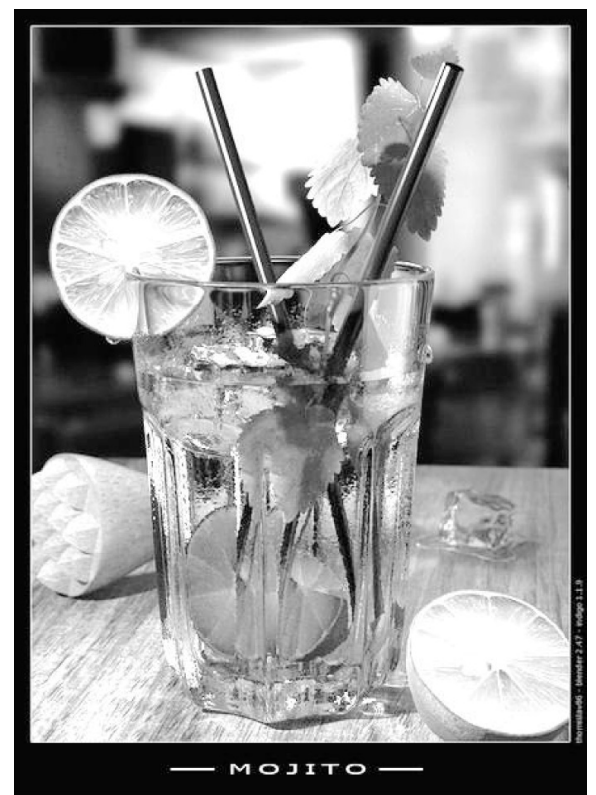

Figura 4. Muestra de Blender.

\begin{tabular}{ll}
\hline 6 & Para mayor información consultar: www.wings3d.com \\
7 & Para mayor información consultar: brlcad.org
\end{tabular}

8 Para mayor información consultar: www.artofillusion.org 


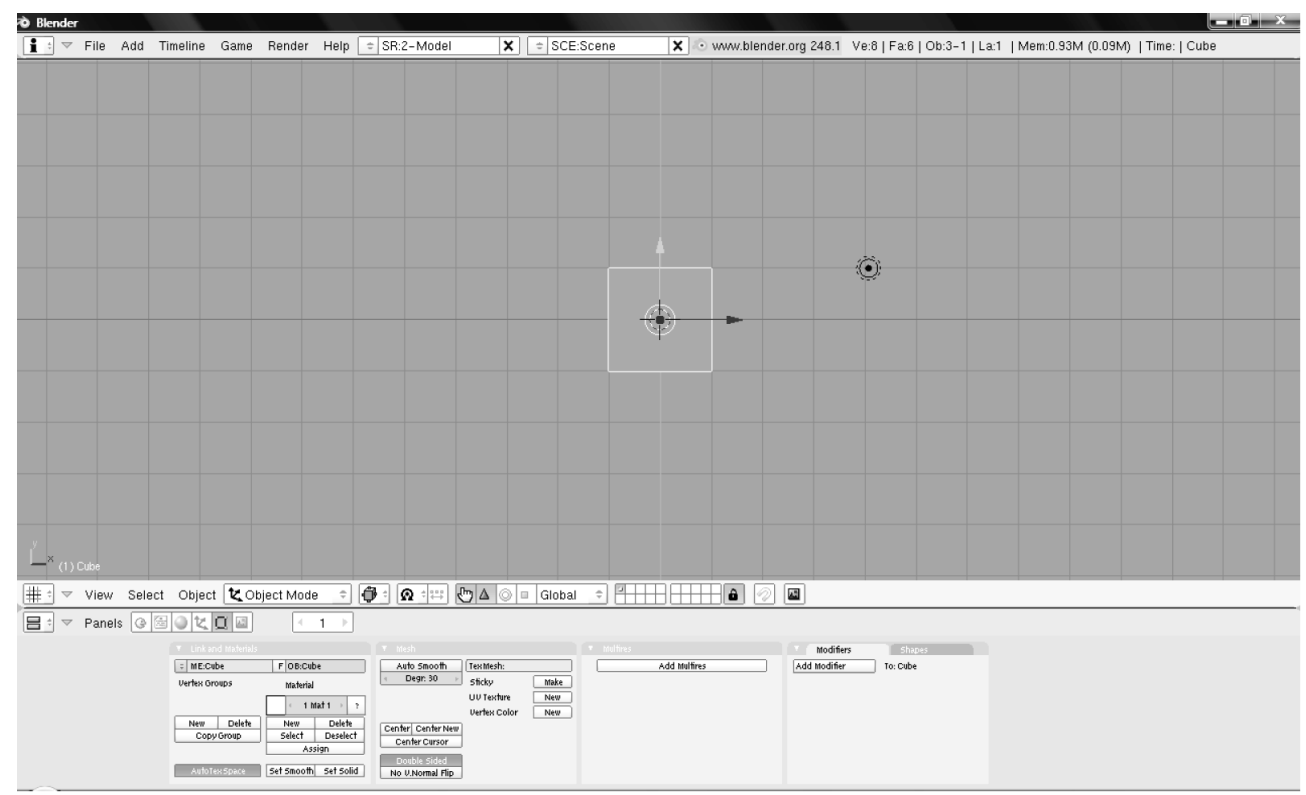

Figura 5. Interfaz de usuario de Blender.

En cambio, y hasta la fecha de publicación del presente artículo, Blender representa quizás la mejor alternativa en este campo; esto debido a la calidad de sus resultados en modelamiento en tres dimensiones, renderizado, movimientos, entre otros. La Figura 4 muestra un resultado de su galería.

Este software puede ser descargado directamente desde su página web en: www.blender.org, tanto su versión ejecutable como su código fuente; ahí el cibernauta podrá verificar que Blender se encuentra reglamentado por la GNU-GPL.

Blender es un programa escrito en código Phyton, el cual también es un software libre de programación, sin embargo, no está dentro de los límites del copyleft ${ }^{9}$, lo que quiere decir que cualquier programa hecho en este lenguaje puede ser utilizado con fines comerciales.

La interfaz de Blender es totalmente distinta a la comúnmente utilizada (es decir a aquellas similares a Microsoft Office), esto puede representar una ventaja y desventaja al mismo tiempo, primeramente porque tiene muchas funciones que hacen más fácil el diseño pero a la vez algunas funciones cotidianas se hacen más difíciles. La Figura 5 muestra la interfaz de este programa.

Para poder facilitar el trabajo con este software, existe una filosofía entre los usuarios de Blender, la cual se puede resumir en la siguiente frase: "Mano derecha en el teclado, mano izquierda en el mouse", esto pues implica manejar una serie de teclas para realizar actividades propias del diseño y al mismo tiempo guiarse con el mouse.

Blender permite la creación de figuras a partir de formas simples llamadas "primitivas" (véase la Figura 6). Debido a que este concepto de creación es muy básico, el programa presenta diversas posibilidades de diseño en el modo EDIT (ver Figura 7). Con respecto al acabado final, este programa puede importar diversas texturas o crear las que uno desee (véase la Figura 8).

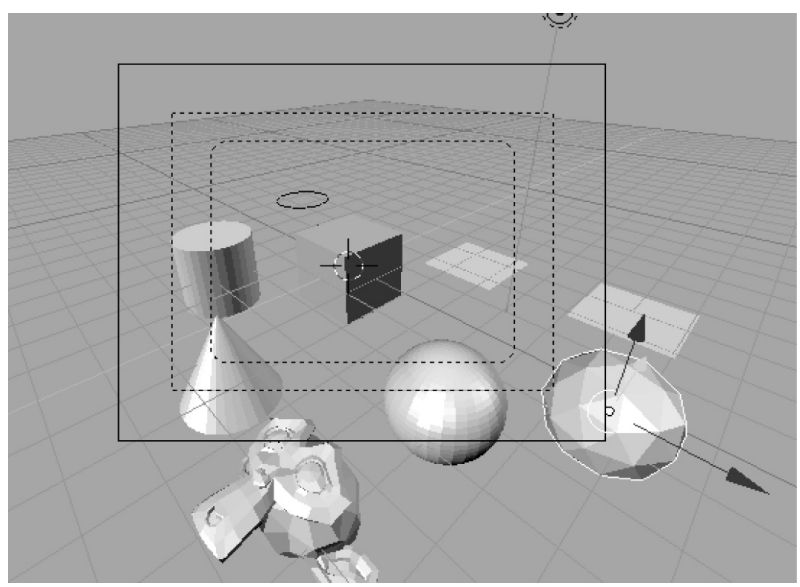

Figura 6. Figuras primitivas de Blender.

En lo que respecta al movimiento, en Blender se pueden realizar diversas animaciones aplicadas al diseño industrial, como por ejemplo la animación

9 Para mayor información consultar: www.phyton.org 
de un ensamble por explosión o también un paseo virtual por alguna instalación en su fase de diseño.

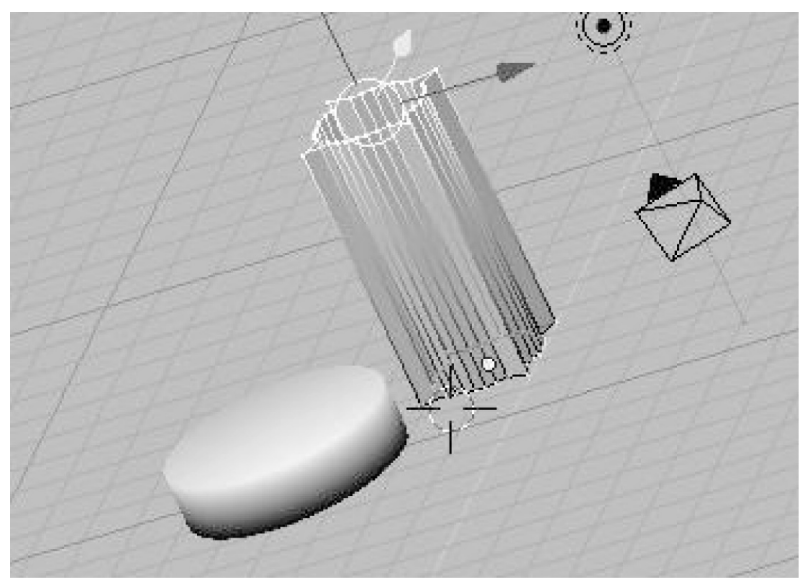

Figura 7. Diseñando en modo EDIT.

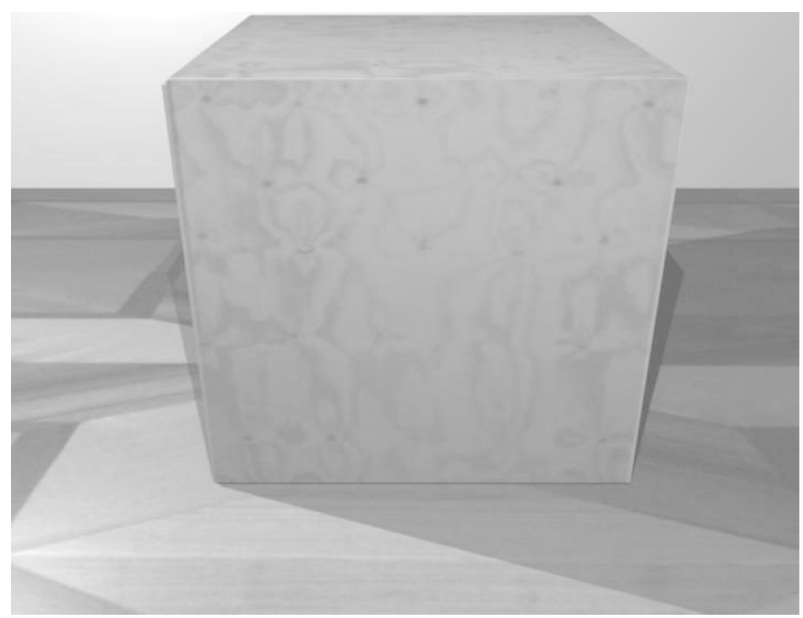

Figura 8. Muestra de texturas hecha en clase.

\section{VENTAJAS Y DESVENTAJAS CON RESPECTO AL SOFTWARE COMERCIAL}

En cuanto a las ventajas que presenta Blender con respecto al software comercial (Inventor, Rhinoceros, 3d Studio etc.), se pueden mencionar las siguientes:

a. La calidad del modelo final es igual o superior a muchos softwares comerciales, lo mismo sucede con las animaciones.

b. No tiene un costo por uso ni por licencia, es decir puede ser descargada la versión completa desde su página web.

c. Puede modificar el software a sus necesidades, porque también está disponible el código fuente.

d. Puede ser actualizado sin costo alguno. e. Tiene una herramienta de Armadura, la cual de la mayor naturalidad a los movimientos humanos.

f. Tiene un potente motor para juegos.

Sin embargo, también presenta algunas desventajas con respecto a los softwares comerciales, entre ellas se pueden mencionar las siguientes:

a. No realiza funciones de simulación.

b. La interfaz de usuario no es muy amigable al inicio, aunque esta desventaja puede ser superada practicando en dicho software.

c. No trabaja con Ingeniería Inversa.

d. Requiere aprenderse varias combinaciones de teclas.

A pesar de estas desventajas, las ventajas que ofrece con respecto al software comercial son muy importantes, lo cual hace de este software (como se dijo anteriormente) una de las alternativas mayormente usadas por los diseñadores.

\section{IMPLEMENTACIÓN DE BLENDER EN UN LABO- RATORIO DE DIBUJO INDUSTRIAL}

Como se explicó anteriormente la instalación de Blender es bastante sencilla. Basta con descargar el instalador correspondiente de su página web. El mayor problema con el que se enfrentará todo estudiante que desee aprender este software será acostumbrarse a su interfaz de usuario; para esto se recomienda dedicar dos o tres clases a familiarizarse con dicha interfaz.

Para la estructuración de las clases en Blender se recomiendan los siguientes puntos en el orden establecido:

1. Presentación del interfaz inicial y de las teclas de funciones básicas.

2. Diseño con figuras primitivas.

3. Diseño en el modo EDIT

4. Aplicación de texturas

5. Creación de animaciones

6. Creación de paseos virtuales

La Figura 9 presenta un ejemplo de lo que un alumno podría hacer como ejercicio en un examen.

Finalmente, cabe mencionar que la respuesta del alumnado de la Facultad de Ingeniería Industrial en los ciclos 2008-I y 2008-Il a este programa ha sido bastante satisfactoria, esto se ha reflejado tanto en sus notas como en los comentarios recogidos en clase; incluso se han generado los llamados comúnmente "blendermaníacos", esto debido a la 
posibilidad de poder crear diversos mundos imaginarios y también a la cantidad de tutoriales que existen en la red acerca de este programa.

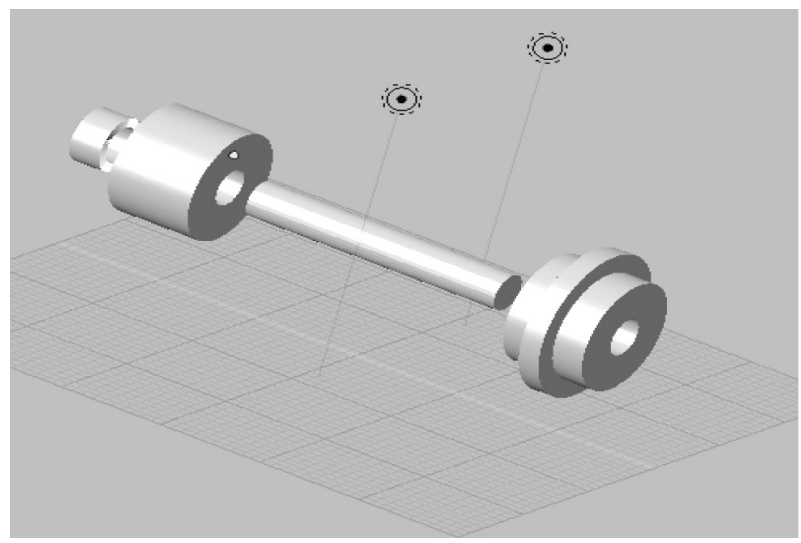

Figura 9. Ejercicio recomendado.

\section{CONCLUSIONES Y RECOMENDACIONES}

Como conclusiones se pueden mencionar las siguientes:

1. Blender representa una alternativa viable en software libre ante softwares comerciales de modelamiento y movimiento.

2. En general, el software libre es una alternativa ante los altos costos del software comercial.

3. El pago de licencias por uso del software comercial tiene un carácter ambivalente, si bien representa el justo pago por el uso de una propiedad intelectual al mismo tiempo es un factor de exclusión de individuos e instituciones al acceso del conocimiento y la tecnología.

4. Si el conocimiento es poder, el software libre ayuda a la democratización de ese poder.

5. Los alumnos asistentes a la implementación del software mostraron una respuesta favorable al aprendizaje de Blender, luego de familiarizarse con su interfaz y de comprender las posibilidades que este programa ofrece.

Al mismo tiempo se recomienda incentivar tanto en docentes como en alumnos la investigación de alternativas en software libre, formando así una red de intercambio de información como se suele trabajar en los temas de software libre.

\section{REFERENCIAS BIBLIOGRÁFICAS}

1. Art of Illusion (2008). Art of Illusion Web Page. http://www.artofillusion.org. (visitado el 15-122008).

2. BRLCAD (2008). BRLCAD - Home. http:brlcad. org. (visitado el 15-12-2008).

3. Open Office (2008). Open Office Home. http:// www.openoffice.org (visitado el 14-12-2008).

4. Proyecto GNU (2008). Proyecto GNU. http:// www.gnu.org (visitado el 15-12-2008).

5. Proyecto MOODLE (2008). Proyecto MOODLE. http://moodle.org (visitado el 14-12-2008).

6. Rojas, O. (2005). Dibujo asistido por computador en la Facultad de Ingeniería Industrial. Industrial Data. Vol. 8, N 1: 18-24.

7. Wings 3D (2008). Wings - Home. http://www. wings3d.com (visitado en 16-12-2008).

8. Proyecto Phyton (2008). Phyton - Home. http:// www.phyton.org. (visitado el 16-12-2008).

9. Proyecto Blender (2008). Blender - Home. http:// www.blender.org. (visitado el 16-12-2008). 